\title{
STATUS OF AGN UNIFICATION MODELS
}

\author{
P. D. BARTHEL \\ Kapteyn Astronomical Institute \\ P.O. Box 800 \\ 9700 AV Groningen \\ NETHERLANDS
}

\section{Introduction}

Unification of Active Galactic Nuclei is obviously multidimensional. Dimensions include the mass and angular momentum of the postulated central black hole, the mass accretion rate through the accretion disk surrounding the black hole, and the orientation of this central engine plus associated dust with respect to the line of sight (e.g., Blandford 1990). As for orientation related unification, considerable progress has been made over the past decade. This review will deal with this geometric unification.

\subsection{MECHANISMS, STANDARD INGREDIENTS}

Mechanisms in AGN include:

1. relativistic beaming in radio jets (as manifested by rapid variability from radio to $\gamma$-rays, superluminal motion, synchrotron self-Compton deficit, ....);

2. emission by an optically thick, non-spherical body (e.g., hot accretion disk);

3. external collimation and shadowing by an anisotropic dust distribution (as manifested by the existence of hidden continua, observed through polarization or photon deficit in extended emission line regions, and hidden broad line regions, observed through polarization or in the less opaque near infrared part of the spectrum).

These anisotropic mechanisms introduce selection biases, they lead to incorrect inferred luminosities and make us overestimate the AGN diversity. They furthermore imply unification at some level. Bringing these dif- 
ferent mechanisms together it appears possible to unify various classes of AGN through orientation. Geometric unification schemes are fun for the proponents as it is a challenging phenomenological exercise to devise such schemes, and are fun for the opponents as it is a challenging exercise to find their weak points.

The standard ingredients of AGN unification schemes are:

1. An opaque dust 'torus' surrounding the AGN continuum source (hot accretion disk) and broad emission line region in the (disk) equatorial plane. This dust configuration creates a wide cone. The aspect angle determines whether the observer sees the continuum and broad lines (Type 1), or not (Type 2). In addition may be present:

2. Two-sided (relativistic) jets emerging along the symmetry (polar) axis of the torus plus accretion disk configuration. Such jets are present in radio loud objects and lead to 'turbo'-type objects, creating a narrow beaming cone among the Type 1's when observed at sufficiently small aspect angles. However, the presence of jets does NOT require the presence of a dusty torus: plain 'turbo'-objects are also possible.

Unification theories have used these ingredients to explain AGN properties. Excellent recent accounts dealing with all aspects of these theories can be found in Antonucci (1993) and in several contributions to the Proceedings of the First Stromlo Symposium 'The Physics of Active Galaxies' (1994, ASP Conference Series vol. 54).

\subsection{BASIC SCHEMES}

The following basic unification schemes are currently fashionable, for (A) radio quiet $A G N$, and (B) radio loud $A G N$.

(A) As for the radio quiet population, aspect effects of ingredient 1 can be responsible for the appearance of a Seyfert galaxy as Type 1 or type 2. There may be a high (optical) luminosity analogue, where radio quiet QSOs (Type 1) have their obscured (Type 2) counterparts among Ultraluminous infrared galaxies (ULIRGs). In addition, nonrelativistic thermal gas outflow may occur in radio quiet QSOs through a limited solid angle. Observed through this solid angle the QSO may reveal Broad Absorption Line troughs.

(B) Turning to the radio loud populations one needs to discriminate 
between the moderately radio luminous objects, the edge-darkened radio galaxies of Fanaroff \& Riley type I, and the highly radio luminous objects, the edge brightened (hotspots) radio galaxies and quasars of Fanaroff \& Riley type II. There are clear physical differences between these two classes of radio sources, and it is noteworthy that FRI quasars do not exist. Hence, observations do not require the presence of either an obscuring dusty torus or broad emission line region (or both) in galaxies associated with FRI radio sources (e.g., Vir A (M87) or Cen A). However, when viewed down the radio jet, an FRI radio galaxy may reveal itself as a BLLac object. The powerful FRII radio sources on the other hand are believed to have both obscuring tori and relativistic jets. Hence the scheme to unify double lobed narrow line radio galaxies such as Cyg $\mathrm{A}$ with lobe dominated (broad line) quasars, which in turn will be observed as core dominated, variable quasars such as $3 \mathrm{C} 345$ when observed down their jets.

Prime question is: are these schemes universal? For example, does every Seyfert 2 galaxy harbour a hidden broad line region? Does every radio-quiet QSO have an intrinsic BAL flow? Does every FRI radio galaxy have relativistic jets? Does every FRII radio galaxy harbour an obscured quasar? In order to address these issues, one needs to make elaborate consistency checks for the various classes of objects, and try to observe the hidden radiation in key objects. To do so properly, we need orientation invariants as well as orientation indicators.

\subsection{PHILOSOPHICAL REMARK}

As mentioned in the introduction, the mere presence of aspect dependent radiation precludes unification of different types of objects. The occurrence of beamed emission in blazars is inescapable ( $\gamma$-rays!); therefore some form of unification of these objects with seemingly different objects has to hold. 'There must be parents for beamed or otherwise favourably oriented objects' is a dogma of the Unification Church.

\section{Healthy schemes}

Unification schemes in good health are, in the eyes of this reviewer:

(i) the Seyfert Type 1 - Type 2 scheme;

(ii) the BLLac - FRI radio galaxy scheme;

(iii) the quasar - FRII narrow line radio galaxy scheme.

However, it is not yet clear how general these schemes are. Moreover, to explain the unification scheme transition with increasing radio or optical 
luminosity is an even greater challenge! As it is impossible to deal with all pro's and con's of these schemes here, I will restrict the discussion to some recent key results, and highlight some well studied key objects.

\subsection{THE SEYFERT UNIFIED SCHEME}

It should be noted that evidence for a hidden continuum in the Seyfert 2 galaxy NGC1068 was already inferred from IUE spectra in the early eighties (Neugebauer et al. 1980). HST images have now revealed its [OIII] ionization cone; it is likely that the radio core of NGC1068 is located at the apex of this cone (Evans et al. 1991). HST spectrolarimetry has measured wavelength independent optical polarization, hence electron scattering, with broad CIII] and MgII emission lines (Antonucci et al. 1994a).

Several nice examples of biconical extended emission line regions have been found with HST (e.g., Wilson et al. 1993) General result from these HST studies is that the emission line regions in Type 1 Seyferts are typically smaller than in Type 2's (Evans et al. 1994). For Seyfert 2's with ionization cones, the predicted reradiated far infrared emission (as inferred from the extended emission lines and torus solid angle coverage) is indeed measured by IRAS (Storchi-Bergmann et al. 1992).

Neutral hydrogen was detected outside the beautiful ionization cones in the Seyfert 2 galaxy NGC5252 (Prieto \& Freudling 1994).

For the CfA Seyfert sample the long standing issue of the radio luminosities of the Type 1's vs. Type 2's is being sorted out: whereas the radio morphologies differ in a sense as predicted by the unified scheme, the radio luminosity distributions are indeed similar (Kukula et al. 1994). The space densities for the CfA Seyfert galaxies differ by a factor $\sim 2.5$, which is similar to the value determined for the $12 \mu$ selected sample (Spinoglio \& Malkan 1989).

Near infrared (less extinction) spectroscopy has revealed broad $\mathrm{Pa} \beta$ lines in several Seyfert 2's (Ruiz et al. 1994). Spectropolarimetry has revealed scattered broad line regions in several Seyfert 2's, but not in all (Kay 1994).

Concluding, the evidence is building up that a fair fraction of Seyfert 2 galaxies harbours hidden Seyfert 1 nuclei. Generalizability of this unified scheme is not clear yet; there may well be two types of Seyfert 2's. Proper jobs on well understood samples such as the CfA sample and the $12 \mu$ sample need to be carried out. 


\subsection{THE FRI UNIFIED SCHEME}

The extended, unbeamed radio luminosity around BLLac objects classifies these objects among the intermediate luminosity Fanaroff \& Riley Class I radio sources. Since BLLac itself is a superluminal source and beaming effects in BLLac objects are a defining property, this unified scheme predicts relativistic jets at parsec scales in FRI radio galaxies. The prediction is indeed borne out by recent VLBI imaging studies of these radio galaxies (e.g., Venturi et al. 1994).

The observed radio luminosity functions are in agreement with the scheme, adopting an average jet Lorentz factor $\langle\gamma\rangle \sim 7$, and average orientation $\langle\phi\rangle \sim 7^{\circ}$ for the radio selected BLLac's. X-ray selected BLLac's have lower $\langle\gamma\rangle$ and consequently wider cones (e.g., Urry et al. 1991).

The NE emission line filaments in Centaurus A need a source of ionization which is not seen directly (Morganti et al. 1992); both a narrow photon beam or shock ionization could be responsible.

HST images have revealed a dusty disk of about 100 parsec dimension perpendicular to the radio axis of the FRI radio galaxy $3 \mathrm{C} 270$, associated with NGC4261 (Jaffe et al. 1993). It should be noted however that such a disk was not seen in M87, and is not required by the scheme (there are no broad line FRI objects).

Again, the generalizability of this scheme is not clear. It is not certain that all FRI radio galaxies have relativistic jets (at small scale), ergo take part in the unified scheme.

\subsection{THE FRII UNIFIED SCHEME}

As even the largest quasars display one-sided jets, superluminal motion, and pronounced depolarization asymmetry, the dogma of Sect. 1.3 is certainly valid here: quasar parents must hide among FRII radio galaxies. In fact, all relationships involving apparent velocities, radio core strength and variability, jet opening angle, overall source projected size and curvature are in the sense as predicted by the scheme.

The scheme is, at least for the most luminous radio sources, broadly consistent with number densities, projected size distributions and luminosity functions, adopting $\langle\gamma\rangle \sim 10$ (Padovani \& Urry 1992). A subpopulation of FRII radio galaxies without hidden BLR is however not ruled out (e.g., Laing et al. 1994)

Radiation anisotropy is particularly apparent in the (FRII) high redshift radio galaxies, which are being systematically studied since the late eighties. Continuum (in particular ultraviolet) and Ly $\alpha$ emission line radiation are generally found to be aligned with the overall, usually double lobed radio 
structure. Both jet induced star formation and scattering of an obscured AGN are proven mechanisms behind this alignment effect (e.g., McCarthy 1993).

Whereas the expected [OIII] extended narrow line emission was expected to be orientation invariant, this expectation proved to be incorrect (Jackson \& Browne 1990). The lower ionization [OII] $\lambda 3727$ line however does seem to classify as such, thereby providing evidence for the FRII scheme (Hes et al. 1993). Similarly, attention was drawn to the fact that the $60 \mu$ far infrared radiation, expected to be orientation invariant, is more luminous in FRII quasars than in (narrow line) radio galaxies (Heckman et al. 1992). This is still a complicated issue. However, for powerful $3 \mathrm{C}$ objects Hes et al. (1994) demonstrate that the inconsistency with the simple FRII scheme may be less severe, pointing out that the $60 \mu$ radiation could be moderately aspect dependent.

A key object for the FRII scheme is obviously Cygnus A, the extremely luminous narrow line radio galaxy in our back yard. Considerable progress has been made examining its properties. Many properties were found to be supportive of the presence of a hidden quasar in the dusty Cyg A nucleus:

- Cyg A has a heavily reddened nuclear narrow emission line region (Vestergaard \& Barthel 1992), and in addition $A_{V} \sim 50^{m}$ towards the nuclear continuum source (Djorgovski et al. 1991, Ward et al. 1991);

- whereas the featureless continuum in the central regions of Cyg A is most likely due to circumnuclear star formation (Stockton et al. 1994), HST has observed broad, polarized MgII in Cyg A, identified as Rayleigh scattered broad line radiation (Antonucci et al. 1994b).

However, despite a good deal of evidence obtained for the highest radio luminosity objects, the generalizability of the FRII scheme is still in question. In particular: does the scheme extent to lower radio power and how can the cosmological size evolution be understood (e.g., Singal 1993).

\section{Aspect effects for luminous radio quiet objects?}

Several cases of ULIRGs were found to display a scattered AGN continuum and/or broad emission lines, hence would classify as Type 2 QSO. This is particularly true for IRAS FSC10214 $+4724(z=2.3)$, where the polarized spectrum indicates a scattered AGN continuum (Jannuzi et al. 1994). It is not at all clear how general this scheme is. Neither is clear if and how the class of BAL QSOs can simply be unified with nonBAL QSOs through aspect effects. 


\section{Future work}

Obviously, more investigations need to be carried out in order to determine the full extent of unification theories. Homogeneous data in all wavelength domains are needed for complete, orientation unbiased samples. Spectropolarimetry at $8 \mathrm{~m}$-class telescopes is eagerly awaited. Observations of isotropic radiation (low frequency radio, far infrared, hard $\mathrm{X}$-rays), as well as models of the radiative transfer properties of dusty tori will be important.

\section{References}

Antonucci, R.: 1993, ARAA 31, 473

Antonucci, R., Hurt, T., Miller, J.: 1994a, ApJ 430, 210

Antonucci, R., Hurt, T., Kinney, A.: 1994b, Nature 371, 313

Blandford, R.D.: 1990, in Saas-Fee Advanced Course 20: Active Galactic Nuclei, Courvoisier \& Mayor, eds. (Springer), 161

Djorgovski, S., et al.: 1991, ApJ 372, L67

Evans, I.N., et al.: 1991, ApJ 369, L27

Evans, I.N., et al.: 1994, in The First Stromlo Symposium: The Physics of Active Galaxies, Bicknell, Dopita, Quinn, eds., ASP Conf. Series 54, 3

Heckman, T.M., Chambers, K.C., Postman, M.: 1992, ApJ 391, 39

Hes, R., Barthel, P.D., Fosbury, R.A.E.: 1993, Nature 326, 362

Hes, R., Barthel, P.D., Hoekstra, H.: 1994, submitted

Jackson, N., Browne, I.W.A.: 1990, Nature 343, 43

Jaffe, W., et al.: 1993, Nature 364, 213

Jannuzi, B.T., et al: 1994, ApJ 429, L49

Kay, L.: 1994, ApJ 430, 196

Kukula, M.J., et al.: 1994, in IAU Symposium 159: Multi-wavelength Continuum Emission of AGN, Couvoisier \& Blecha, eds., (Dordrecht: Reidel), 514

Laing, R.A., et al.: 1994, in The First Stromlo Symposium: The Physics of Active Galaxies, Bicknell, Dopita, Quinn, eds., ASP Conf. Series 54, 201

McCarthy, P.J.: 1993, ARAA 31, 639

Morganti, R., et al.: 1992, MNRAS 256, 1P

Neugebauer, G., et al.: 1980, ApJ 238, 502

Padovani, P., Urry, C.M.: 1992, ApJ 387, 449

Prieto, M.A., Freudling, W.: 1994, ApJ 418, 668

Ruiz, M., Rieke, G.H., Schmidt, G.D.: 1994, ApJ 423, 608

Singal, A.K.: 1993, MNRAS 263, 139

Spinoglio, L., Malkan, M.A.: 1989, ApJ 342, 83

Stockton, A., Ridgeway, S.E., Lilly, S.: 1994, AJ 108, 414

Storchi-Bergmann, T., et al.: 1992, ApJ 395, L73

Urry, C.M., Padovani, P., Stickel, M.: 1991, ApJ 382, 501

Venturi, T., et al.: 1994, in The First Stromlo Symposium: The Physics of Active Galaxies, Bicknell, Dopita, Quinn, eds., ASP Conf. Series 54, 247

Vestergaard, M., Barthel, P.D.: 1993, AJ 105, 456

Ward, M.J., et al.: 1991, ApJ 382, 115

Wilson, A.S., et al.: 1993, ApJ 419, L61 